\title{
Hemi-Slant Warped Product Submanifolds of Nearly Kaehler Manifolds
}

\author{
Falleh R. Al-Solamy ${ }^{1}$ and Meraj Ali Khan ${ }^{2}$ \\ ${ }^{1}$ Department of Mathematics, King Abdulaziz University, P.O. Box 80015, Jeddah 21589, Saudi Arabia \\ ${ }^{2}$ Department of Mathematics, University of Tabuk, Tabuk, Saudi Arabia
}

Correspondence should be addressed to Meraj Ali Khan; meraj79@gmail.com

Received 25 January 2014; Revised 16 April 2014; Accepted 24 April 2014; Published 12 May 2014

Academic Editor: Irena Rachůnková

Copyright (C) 2014 F. R. Al-Solamy and M. A. Khan. This is an open access article distributed under the Creative Commons Attribution License, which permits unrestricted use, distribution, and reproduction in any medium, provided the original work is properly cited.

\begin{abstract}
Hemi-slant warped product submanifolds of nearly Kaehler manifolds are studied and some interesting results are obtained. Moreover, an inequality is established for squared norm of second fundamental form and equality case is also discussed. The results obtained are also true if ambient manifold is replaced by a Kaehler manifold. These results generalize several known results in the literature.
\end{abstract}

\section{Introduction}

In [1] Bishop and O’Neill introduced the notion of warped product manifolds as a natural generalization of Riemannian product manifolds. For instance, a surface of revolution is a warped product manifold. So far as its applications are concerned, it has been shown that warped product manifolds provide excellent setting to model space time near black holes or bodies with large gravitational forces (see $[1,2]$ ). Due to wide applications of warped product submanifolds, this becomes a fascinating and interesting topic for research and many articles are available in literature (see $[1,3-5]$ ). Chen [6] initiated the study of warped product submanifolds by showing that there do not exist warped product CR-submanifolds of the type $N_{\perp} \times{ }_{f} N_{T}$, and he considered warped product CR-submanifolds of the types $N_{T} \times{ }_{f} N_{\perp}$ and established a relationship between the warping function $f$ and the squared norm of the second fundamental form. Extending the study of Chen, Sahin [7] proved that there exist no semislant warped product submanifolds in a Kaehler manifold. In [8], V. A. Khan and K. A. Khan studied generic warped product submanifolds of nearly Kaehler manifolds and obtained an inequality for squared norm of second fundamental form in terms of warping function. Recently, Sahin [4] investigated hemi-slant warped product for Kaehler manifolds and obtained an inequality for squared norm of second fundamental form for mixed totally geodesic submanifolds. In view of the interesting geometric characteristic of nearly Kaehler manifolds and the nonexistence of CRproduct submanifolds in $S^{6}$ [9], it will be significant to explore hemi-slant warped product submanifolds of a nearly Kaehler manifold. In this continuation we have achieved success in extending the results of Sahin [4] and Chen [6] to the setting of nearly Kaehler manifolds.

\section{Preliminaries}

Let $(\bar{M}, J, g)$ be a nearly Kaehler manifold with an almost complex structure $J$ and Hermitian metric $g$ and a Levi-Civita connection $\bar{\nabla}$ such that

$$
\begin{gathered}
g(J X, J Y)=g(X, Y), \\
\left(\bar{\nabla}_{X} J\right) Y+\left(\bar{\nabla}_{Y} J\right) X=0,
\end{gathered}
$$

for all vector fields $X$ and $Y$ on $\bar{M}$. Six-dimensional sphere $S^{6}$ is a classic example of a nearly Kaehler non-Kaehler manifold. It has an almost complex structure $J$ defined by the vector cross product in the space of purely imaginary Cayley numbers which satisfies the condition $\left(\bar{\nabla}_{X} J\right) X=0$. Let $C$ be the Cayley division algebra generated by $\left\{e_{0}=1, e_{i}\right.$, $(1 \leq i \leq 7)\}$ over $R$ and $C_{+}$the subspace of $C$ consisting 
of all purely imaginary Cayley numbers. We may identify $C_{+}$ with a 7-dimensional Euclidean space $R^{7}$ with the canonical inner product $g=($, ). The automorphism group of $C$ is the compact simple Lie group $G_{2}$ and furthermore the inner product $g$ is invariant under the action of $G_{2}$ and hence, the group $G_{2}$ may be considered as a subgroup of $S O(7)$. A vector cross product for vectors in $R^{7}\left(=C_{+}\right)$is defined by

$$
x \times y=(x, y) e_{0}+x y, \quad \forall x, y \in C_{+} .
$$

Then the multiplication table for $e_{j} \times e_{k}$ is given by

\begin{tabular}{c|ccccccc}
$\frac{j}{k}$ & 1 & 2 & 3 & 4 & 5 & 6 & 7 \\
\hline 1 & 0 & $e_{3}$ & $-e_{2}$ & $e_{5}$ & $-e_{4}$ & $e_{7}$ & $-e_{6}$ \\
2 & $-e_{3}$ & 0 & $e_{1}$ & $e_{6}$ & $-e_{7}$ & $-e_{4}$ & $e_{5}$ \\
3 & $e_{2}$ & $-e_{1}$ & 0 & $-e_{7}$ & $-e_{6}$ & $e_{5}$ & $e_{4}$ \\
4 & $-e_{5}$ & $-e_{6}$ & $e_{7}$ & 0 & $e_{1}$ & $e_{2}$ & $-e_{3}$ \\
5 & $e_{4}$ & $e_{7}$ & $e_{6}$ & $-e_{1}$ & 0 & $-e_{3}$ & $-e_{2}$ \\
6 & $-e_{7}$ & $e_{4}$ & $-e_{5}$ & $-e_{2}$ & $e_{3}$ & 0 & $e_{1}$ \\
7 & $e_{6}$ & $-e_{5}$ & $-e_{4}$ & $e_{3}$ & $e_{2}$ & $-e_{1}$ & 0
\end{tabular}

Considering $S^{6}$ as $\left\{x \in C_{+}:(x, x)=1\right\}$, the almost complex structure $J$ on $S^{6}$ is defined by

$$
J_{x}(X)=x \times X
$$

where $x \in S^{6}$ and $X \in T_{x} S^{6}$. The almost complex structure defined by the above equation together with the induced metric on $S^{6}$ from $g$ on $R^{7}\left(=C_{+}\right)$gives rise to a nearly Kaehler structure $S^{6}[10]$.

Let $M$ be a submanifold of $\bar{M}$. Then the induced Riemannian metric on $M$ is denoted by the same symbol $g$ and the induced connection on $M$ is denoted by the symbol $\nabla$. If $T \bar{M}$ and $T M$ denote the tangent bundle on $\bar{M}$ and $M$, respectively, and $T^{\perp} M$, the normal bundle on $M$, then the Gauss and Weingarten formulae are given by

$$
\begin{aligned}
& \bar{\nabla}_{X} Y=\nabla_{X} Y+h(X, Y), \\
& \bar{\nabla}_{X} N=-A_{N} X+\nabla_{X}^{\perp} N,
\end{aligned}
$$

for $X, Y \in T M$ and $N \in T^{\perp} M$ where $\nabla^{\perp}$ denotes the connection on the normal bundle $T^{\perp} M . h$ and $A_{N}$ are the second fundamental form and the shape operator of immersions of $M$ into $\bar{M}$. Corresponding to the normal vector field $V$ they are related as

$$
g\left(A_{V} X, Y\right)=g(h(X, Y), V) .
$$

The mean curvature vector $H$ of $M$ is given by

$$
H=\frac{1}{n} \sum_{i=1}^{n} h\left(e_{i}, e_{i}\right),
$$

where $n$ is the dimension of $M$ and $\left\{e_{1}, e_{2}, \ldots, e_{n}\right\}$ is a local orthonormal frame of vector fields on $M$. The squared norm of the second fundamental form is defined as

$$
\|h\|^{2}=\sum_{i, j=1}^{n} g\left(h\left(e_{i}, e_{j}\right), h\left(e_{i}, e_{j}\right)\right) .
$$

A submanifold $M$ of $\bar{M}$ is said to be a totally geodesic submanifold if $h(X, Y)=0$ for each $X, Y \in T M$, and totally umbilical submanifold if $h(X, Y)=g(X, Y) H$.

For $X \in T M$ and $N \in T^{\perp} M$ we write

$$
\begin{aligned}
& J X=P X+F X, \\
& J N=t N+f N,
\end{aligned}
$$

where $P X$ and $t N$ are tangential components of $J X$ and $J N$, respectively, and $F X$ and $f N$ are the normal components of $J X$ and $J N$.

The covariant differentiation of the tensors $P, F, t$, and $f$ is defined, respectively, as

$$
\begin{gathered}
\left(\bar{\nabla}_{X} P\right) Y=\nabla_{X} P Y-P \nabla_{X} Y, \\
\left(\bar{\nabla}_{X} F\right) Y=\nabla_{X}^{\perp} F Y-F \nabla_{X} Y, \\
\left(\bar{\nabla}_{X} t\right) N=\nabla_{X} t N-t \nabla_{X}^{\perp} N, \\
\left(\bar{\nabla}_{X} f\right) N=\nabla_{X}^{\perp} f N-f \nabla_{X}^{\perp} N .
\end{gathered}
$$

Furthermore, for any $X, Y \in T M$, the tangential and normal parts of $\left(\bar{\nabla}_{X} J\right) Y$ are denoted by $\mathscr{P}_{X} Y$ and $\mathbb{Q}_{X} Y$; that is,

$$
\left(\bar{\nabla}_{X} J\right) Y=\mathscr{P}_{X} Y+Q_{X} Y
$$

On using (6)-(13), we may obtain that

$$
\begin{gathered}
\mathscr{P}_{X} Y=\left(\bar{\nabla}_{X} P\right) Y-A_{F Y} X-\operatorname{th}(X, Y), \\
Q_{X} Y=\left(\bar{\nabla}_{X} F\right) Y+h(X, T Y)-f h(X, Y) .
\end{gathered}
$$

Similarly, for $N \in T^{\perp} M$, denoting by $\mathscr{P}_{X} N$ and $\mathscr{Q}_{X} N$, respectively, the tangential and normal parts of $\left(\bar{\nabla}_{X} J\right) N$, we find that

$$
\begin{gathered}
\mathscr{P}_{X} N=\left(\bar{\nabla}_{X} t\right) N+P A_{N} X-A_{f N} X, \\
\mathcal{Q}_{X} N=\left(\bar{\nabla}_{X} f\right) N+h(t N, X)+F A_{N} X .
\end{gathered}
$$

On a submanifold $M$ of a nearly Kaehler manifold by (2) and (16)
(a) $\mathscr{P}_{X} Y+\mathscr{P}_{Y} X=0$,
(b) $\mathbb{Q}_{X} Y+Q_{Y} X=0$

for any $X, Y \in T M$.

Let $\bar{M}$ be an almost Hermitian manifold with an almost complex structure $J$ and Hermitian metric $g$ and let $M$ be a submanifold of $\bar{M}$. Submanifold $M$ is said to be CRsubmanifold if there exist two orthogonal complementary distributions $D$ and $D^{\perp}$ such that $D$ is holomorphic distribution, that is, $J D \subseteq D$ and $D^{\perp}$ is totally real distribution, that is, $J D^{\perp} \subseteq T^{\perp} M$.

An immersed submanifold $M$ of an almost Hermitian manifold $\bar{M}$ is said to be slant submanifold if the Wirtinger angle $\theta(X) \in[0, \pi / 2]$ between $J X$ and $T_{x} M$, and $X \neq 0$ (cf. [11]). Holomorphic and totally real submanifolds are slant submanifolds with wirtinger angle 0 and $\pi / 2$. 
A submanifold is called proper slant if it is neither holomorphic nor totally real. More generally, a distribution $D_{\theta}$ on $\bar{M}$ is called a slant distribution if the angle $\theta(X)$ between $J X$ and $D_{\theta}$ has the same value $\theta$ for each $x \in \bar{M}$ and $X \in D_{\theta}$ and $X \neq 0$.

If $M$ is a slant submanifold of an almost Hermitian manifold $\bar{M}$, then we have (cf. [11])

$$
P^{2} X=-\cos ^{2} \theta X
$$

where $\theta$ is the wirtinger angle of $M$ in $\bar{M}$. Hence we have

$$
\begin{aligned}
& g(P X, P Y)=\cos ^{2} \theta g(X, Y), \\
& g(F X, F Y)=\sin ^{2} \theta g(X, Y),
\end{aligned}
$$

for any $X, Y \in T M$.

Papaghiuc [12] introduced a class of submanifolds in almost Hermitian manifolds called the semislant submanifolds; this class includes the class of proper CR-submanifolds and slant submanifolds. Cabrerizo et al. [13] initiated the study of contact version of semislant submanifold and also bislant submanifolds. As a step forward, Carriazo [14] defined and studied bislant submanifolds and simultaneously gave the notion of antislant submanifolds in almost Hermitian manifolds; after that V. A. Khan and M. A. Khan [15] studied antislant submanifolds with the name pseudo-slant submanifolds in the setting of Sasakian manifolds.

Recently, Sahin [4] renamed pseudo-slant submanifolds as hemi-slant submanifolds and studied hemi-slant submanifolds for their warped product.

Definition 1. A submanifold $M$ of an almost Hermitian manifold is called hemi-slant submanifold if it is endowed with two orthogonal complementary distributions $D^{\perp}$ and $D_{\theta}$ such that $D^{\perp}$ is totally real, that is, $J D^{\perp} \subseteq T^{\perp} M$ and $D_{\theta}$ is slant distribution with slant angle $\theta$.

It is straight forward to see that CR-submanifolds and slant submanifolds are hemi-slant submanifolds with $\theta=0$ and $D^{\perp}=\{0\}$, respectively.

If $\mu$ is the invariant subspace of the normal bundle $T^{\perp} M$ then in the case of hemi-slant submanifold, the normal bundle $T^{\perp} M$ can be decomposed as follows:

$$
T^{\perp} M=\mu \oplus F D^{\perp} \oplus F D_{\theta} .
$$

As $D^{\perp}$ and $D_{\theta}$ are orthogonal distributions on $M$, then it is easy to see that the distributions $F D^{\perp}$ and $F D_{\theta}$ are mutually perpendicular. In fact, the decomposition (24) is an orthogonal direct decomposition.

A hemi-slant submanifold $M$ is called a hemi-slant product if the distributions $D^{\perp}$ and $D_{\theta}$ are parallel on $M$. In this case $M$ is foliated by the leaves of these distributions. In particular if $M$ is CR-submanifold with parallel distribution then it is called CR-product. In general, if $M_{1}$ and $M_{2}$ are Riemannian manifolds with Riemannian metrics $g_{1}$ and $g_{2}$, respectively, then the product manifold $\left(M_{1} \times M_{2}, g\right)$ is a Riemannian manifold with Riemannian metric $g$ defined as

$$
g(X, Y)=g_{1}\left(d \pi_{1} X, d \pi_{1} Y\right)+g_{2}\left(d \pi_{2} X, d \pi_{2} Y\right),
$$

where $\pi_{i}(i=1,2)$ are the projection maps of $M$ onto $M_{1}$ and $M_{2}$, respectively, and $d \pi_{i}(i=1,2)$ are their differentials.

As a generalization of the product manifold and in particular of a hemi-slant product submanifold, one can consider warped product of manifolds which are defined in the following.

Definition 2. Let $\left(B, g_{B}\right)$ and $\left(C, g_{C}\right)$ be two Riemannian manifolds with Riemannian metrics $g_{B}$ and $g_{C}$, respectively, and $f$ a positive differentiable function on $B$. The warped product of $B$ and $C$ is the Riemannian manifold $(B \times C, g)$, where

$$
g=g_{B}+f^{2} g_{C}
$$

For a warped product manifold $N_{1} \times{ }_{f} N_{2}$, we denote by $D_{1}$ and $D_{2}$ the distributions defined by the vectors tangent to the leaves and fibers, respectively. In other words, $D_{1}$ is obtained by the tangent vectors of $N_{1}$ via the horizontal lift and $D_{2}$ is obtained by the tangent vectors of $N_{2}$ via vertical lift. In case of semi-invariant warped product submanifolds $D_{1}$ and $D_{2}$ are replaced by $D$ and $D^{\perp}$, respectively.

The warped product manifold $(B \times C, g)$ is denoted by $B \times{ }_{f} C$. If $X$ is the tangent vector field to $M=B \times{ }_{f} C$ at $(p, q)$ then

$$
\|X\|^{2}=\left\|d \pi_{1} X\right\|^{2}+f^{2}(p)\left\|d \pi_{2} X\right\|^{2} .
$$

Bishop and O'Neill [1] proved the following.

Theorem 3. Let $M=B \times{ }_{f} C$ be warped product manifolds. If $X, Y \in T B$ and $V, W \in T C$ then

(i) $\nabla_{X} Y \in T B$,

(ii) $\nabla_{X} V=\nabla_{V} X=(X f / f) V$,

(iii) $\nabla_{V} W=(-g(V, W) / f) \nabla f$.

$\nabla f$ is the gradient of $f$ and is defined as

$$
g(\nabla f, X)=X f
$$

for all $X \in T M$.

Corollary 4. On a warped product manifold $M=N_{1} \times_{f} N_{2}$, the following statements hold:

(i) $N_{1}$ is totally geodesic in $M$

(ii) $\mathrm{N}_{2}$ is totally umbilical in $M$.

In what follows, $N_{\perp}$ and $N_{\theta}$ will denote a totally real and slant submanifold, respectively, of an almost Hermitian manifold $\bar{M}$.

A warped product manifold is said to be trivial if its warping function $f$ is constant. More generally, a trivial warped product manifold $M=N_{1} \times N_{2}$ is a Riemannian product $N_{1} \times N_{2}^{f}$, where $N_{2}^{f}$ is the manifold with the Riemannian metric $f^{2} g_{2}$ which is homothetic to the original metric $g_{2}$ of $N_{2}$. For example, a trivial CR-warped product is CR-product.

Sahin [4] extended the study of warped product hemislant submanifolds and hemi-slant warped product of Kaehler manifolds introducing warped product submanifolds as $N_{\theta} \times_{f 2} N_{\perp}$ and $N_{\perp} \times_{f} N_{\theta}$, where $\theta$ is the slant angle. 


\section{Hemi-Slant Warped Product Submanifolds}

In [5] Uddin and Chi investigated warped product pseudoslant (hemi-slant) submanifolds of nearly Kaehler manifolds and they only showed that there do not exist warped products of the form $N_{\perp} \times{ }_{f} N_{\theta}$ in nearly Kaehler manifolds, where $N_{\perp}$ is totally real submanifold and $N_{\theta}$ is slant submanifold. In this section we study the warped products of the types $N_{\theta} \times{ }_{f} N_{\perp}$.

Let $M=N_{\theta} \times{ }_{f} N_{\perp}$ be a hemi-slant warped product of a nearly Kaehler manifold $\bar{M}$. Then by Theorem 3,

$$
\nabla_{X} Z=\nabla_{Z} X=X \ln f Z,
$$

for any $X \in T N_{\theta}, Z \in T N_{\perp}$.

Now by formula (12) and Theorem 3 ,

$$
\left(\bar{\nabla}_{Z} P\right) W=g(Z, W) P(\nabla \ln f)-g(Z, P W) \nabla \ln f,
$$

for each $Z, W \in T N_{\theta}$. Now we will investigate some interesting results of the second fundamental form.

Proposition 5. On a hemi-slant warped product submanifold $M=N_{\theta} \times{ }_{f} N_{\perp}$ of a nearly Kaehler manifold $\bar{M}$, one has

(i) $2 g(h(X, Y), F Z)=g(h(X, Z), F Y)+g(h(Y, Z), F X)$,

(ii) $g(h(X, Z), F X)=g(h(X, X), F Z)$,

for any $X, Y \in N_{\theta}$ and $Z \in N_{\perp}$.

Proof. As $N_{\theta}$ is totally geodesic in $M$ then $\left(\bar{\nabla}_{X} P\right) Y \in T N_{\theta}$ and therefore by formula (17),

$$
g\left(\mathscr{P}_{X} Y, Z\right)=-g\left(A_{F X} Y, Z\right)-g(t h(X, Y), Z),
$$

or

$$
g(h(X, Y), F Z)=g\left(\mathscr{P}_{X} Y, Z\right)+g(h(Y, Z), F X) .
$$

Similarly, we have

$$
g(h(X, Y), F Z)=g\left(\mathscr{P}_{Y} X, Z\right)+g(h(X, Z), F Y) .
$$

Adding above two equations and using (20)(a), we get part (i).

Now by formula (17) and (20)(a),

$$
0=\left(\bar{\nabla}_{X} P\right) Z+\left(\bar{\nabla}_{Z} P\right) X-2 \operatorname{th}(X, Z)-A_{F Z} X-A_{F X} Z,
$$

and by (29) the above equation gives

$$
(P X \ln f) Z=2 t h(X, Z)+A_{F Z} X+A_{F X} Z .
$$

Taking inner product of (35) with $X \in D_{\theta}$, we get

$$
g(h(X, Z), F X)=g(h(X, X), F Z),
$$

which proves part (ii).

Theorem 6. For a hemi-slant warped product submanifold $N_{\theta} \times{ }_{f} N_{\perp}$ of a nearly Kaehler manifold the warping function satisfies the following relation:

$$
\cos ^{2} \theta X \ln f\|Z\|^{2}=g(h(P X, Z), F Z)-g(H, F P X)\|Z\|^{2},
$$

for any $X \in T N_{\theta}$ and $Z \in T N_{\perp}$.
Proof. If $M$ is a hemi-slant warped product submanifold $N_{\theta} \times{ }_{f} N^{\perp}$ of a nearly Kaehler manifold then $\left(\bar{\nabla}_{X} P\right) Z=0$ for each $X \in T N_{\theta}$ and $Z \in T N_{\perp}$, and thus by (17),

$$
\mathscr{P}_{X} Z=-A_{F Z} X-\operatorname{th}(X, Z) .
$$

On the other hand

$$
\mathscr{P}_{Z} X=\nabla_{Z} P X-P \nabla_{Z} X-A_{F X} Z-\operatorname{th}(X, Z) .
$$

Now using (29), the above equation takes the form

$$
\mathscr{P}_{Z} X=P X \ln f Z-A_{F X} Z-t h(X, Z) .
$$

Adding (38) and (40) and using (20)(a),

$$
A_{F Z} X+A_{F X} Z=P X \ln f Z-2 t h(X, Z),
$$

taking inner product with $Z \in T N_{\perp}$, and using the fact that $N_{\perp}$ is totally umbilical, one gets the following equation:

$$
P X \ln f\|Z\|^{2}=-g(h(X, Z), F Z)+g(H, F X)\|Z\|^{2} .
$$

By replacing $X$ by $P X$ the required result follows.

Remark 7. In [4] Sahin proved that hemi-slant warped products of the type $N_{\perp} \times{ }_{f} N_{\theta}$ do not exist in the setting of Kaehler manifolds. Therefore, in the following Corollary we discuss the warped products of the type $N_{\theta} \times{ }_{f} N_{\perp}$.

Corollary 8. For a hemi-slant warped product submanifold $N_{\theta} \times{ }_{f} N_{\perp}$ of a Kaehler manifold the warping function satisfies the following relation:

$$
\cos ^{2} \theta X \ln f\|Z\|^{2}=g(h(P X, Z), F Z)-g(H, F P X)\|Z\|^{2},
$$

for any $X \in T N_{\theta}$ and $Z \in T N_{\perp}$.

Proof. Since $M$ is a hemi-slant warped product submanifold of a Kaehler manifold, then by tensorial equation of Kaehler manifold, it is easy to see that $\mathscr{P}_{Z} X=0$, for any $X \in T N_{\theta}$ and $Z \in T N_{\perp}$, and using this fact in (40) and taking inner product with $Z \in T N_{\perp}$, we get the required result.

Let us denote by $D_{\theta}$ and $D^{\perp}$ the tangent bundles on $N_{\theta}$ and $N_{\perp}$, respectively, and let $\left\{X_{1}, X_{2}, \ldots, X_{p}, X_{p+1}=\right.$ $\left.P X_{1}, \ldots, X_{2 p}=P X_{p}\right\}$ and $\left\{Z_{1}, Z_{2}, \ldots, Z_{q}\right\}$ be local orthonormal frames of vector fields on $N_{\theta}$ and $N_{\perp}$, respectively, with $2 p$ and $q$ being their real dimensions; then

$$
\begin{aligned}
\|h\|^{2}= & \sum_{i, j=1}^{2 p} g\left(h\left(X_{i}, X_{j}\right), h\left(X_{i}, X_{j}\right)\right) \\
& +\sum_{i=1}^{2 p} \sum_{r=1}^{q} g\left(h\left(X_{i}, Z_{r}\right), h\left(X_{i}, Z_{r}\right)\right) \\
& +\sum_{r, s=1}^{q} g\left(h\left(Z_{r}, Z_{s}\right), h\left(Z_{r}, Z_{s}\right)\right) .
\end{aligned}
$$

Now we calculate the inequality for the squared norm of second fundamental form in the following theorem. 
Theorem 9. Let $M=N_{\theta} \times{ }_{f} N_{\perp}$ be a hemi-slant warped product of a nearly Kaehler manifold $\bar{M}$ with $N_{\theta}$ and $N_{\perp}$ slant and totally real submanifolds, respectively, of $\bar{M}$. If $H \in \mu$, then

(i) the second fundamental form satisfies the following inequality:

$$
\|h\|^{2} \geq 4 q \cos ^{2} \theta\|\nabla \ln f\|^{2}
$$

(ii) equality holds if $h(D, D)=0, h\left(D^{\perp}, D^{\perp}\right)=0$, $h\left(P X_{i}, Z_{r}\right)$ is normal to $F Z_{t}$ and $h\left(P X_{i}, Z_{r}\right)$ is normal to $F X_{i}$ and $F X_{s}$ where $i, s=1,2, \ldots, 2 p$ and $r, t=$ $1, \ldots, q(i \neq s$ and $r \neq t)$,

where $\nabla \ln f$ is the gradient of $\ln f$ and $q$ is dimension of $N_{\perp}$.

Proof. In view of (24) the second fundamental form can be decomposed as follows:

$$
h(U, V)=h_{F D_{\theta}}(U, V)+h_{F D_{\perp}}(U, V)+h_{\mu}(U, V),
$$

for each $U, V \in T M$, where $h_{F D_{\theta}}(U, V) \in F D_{\theta}, h_{F D_{\perp}}(U, V) \in$ $F D_{\perp}$ and $h_{\mu}(U, V) \in \mu$ with

$$
h_{F D_{\theta}}(U, V)=\sum_{i=1}^{2 p} h^{i}(U, V) F X_{i}
$$

where,

$$
\begin{gathered}
h^{i}(U, V)=\csc ^{2} \theta g\left(h(U, V), F X_{i}\right), \\
h_{F D_{\perp}}(U, V)=\sum_{r=1}^{q} h^{r}(U, V) F Z_{r},
\end{gathered}
$$

where,

$$
h^{r}(U, V)=g\left(h(U, V), F Z_{r}\right) \text {, }
$$

for each $X_{i} \in D_{\theta}$ and $Z_{r} \in D_{\perp}$.

Now, making use of (37) with assumption $H \in \mu$ and formulae (48) and (49), we obtain

$$
\begin{aligned}
g & \left(h_{F D_{\perp}}\left(P X_{i}, Z_{r}\right), h_{F D_{\perp}}\left(P X_{i}, Z_{r}\right)\right) \\
& =\cos ^{2} \theta h^{r}\left(P X_{i}, Z_{r}\right)\left(X_{i} \ln f\right)+\sum_{t \neq r}\left(h^{t}\left(P X_{i}, Z_{r}\right)\right)^{2} .
\end{aligned}
$$

Summing over $i=1, \ldots, 2 p$ and $r=1, \ldots, q$ and (37) and assumption $H \in \mu$ with formula (50) the above equation gives

$$
\begin{aligned}
& g\left(h_{F D_{\perp}}\left(P X_{i}, Z_{r}\right), h_{F D_{\perp}}\left(P X_{i}, Z_{r}\right)\right) \\
& =2 q \cos ^{4} \theta\|\nabla \ln f\|^{2}+\sum_{i=1}^{2 p} \sum_{r, t=1, r \neq t}^{q}\left(h^{t}\left(P X_{i}, Z_{r}\right)\right)^{2} .
\end{aligned}
$$

Let us consider the orthonormal frame of vector fields on $D_{\perp}$ as $\left\{Z_{1}, \ldots, Z_{q}\right\}$, and the second term in the right hand side of last equation on using (50) can be written as

$$
\begin{aligned}
\sum_{i=1}^{2 p}\left[\sum_{r=1}^{q}\left(g\left(h\left(P X_{i}, Z_{r}\right), F Z_{r}\right)\right)^{2}\right. & \\
& \left.+\sum_{r=1}^{q} \sum_{t=1, t \neq r}^{q}\left(g\left(h\left(P X_{i}, Z_{r}\right), F Z_{t}\right)\right)^{2}\right] .
\end{aligned}
$$

On applying (37), the first part of above expression reduced to

$$
\sum_{i=1}^{2 p} \sum_{r=1}^{q}\left(g\left(h\left(P X_{i}, Z_{r}\right), F Z_{r}\right)\right)^{2}=2 q \cos ^{4} \theta\|\nabla \ln f\|^{2} .
$$

Taking account of the above equation into (52), we obtain

$$
g\left(h_{F D_{\perp}}\left(P X_{i}, Z_{r}\right), h_{F D_{\perp}}\left(P X_{i}, Z_{r}\right)\right) \geq 4 q \cos ^{4} \theta\|\nabla \ln f\|^{2} .
$$

The inequality follows from (44) and (55).

To discuss the equality case we will explore the expression $\left\|h_{F D_{\theta}}\left(P X_{i}, Z_{r}\right)\right\|^{2}$ as follows.

Making use of (47), (48), and (23) and summing over $r=$ $1, \ldots, q$ and $i=1, \ldots, 2 p$ we find

$$
\begin{aligned}
g\left(h_{F D_{\theta}}\left(P X_{i}, Z_{r}\right), h_{F D_{\theta}}\left(P X_{i}, Z_{r}\right)\right) & \\
=\sin ^{2} \theta \sum_{r=1}^{q}\left[\left\{\sum_{i=1}^{2 p}\left(h^{i}\left(P X_{i}, Z_{r}\right)\right)^{2}\right.\right. & \\
& \left.\left.+\sum_{i, s=1, s \neq i}^{2 p}\left(h^{s}\left(P X_{i}, Z_{r}\right)\right)^{2}\right\}\right] .
\end{aligned}
$$

Let us choose the orthonormal frame of vectors fields on $D_{\theta}$ as $\left\{X_{1}, \ldots, X_{p}, P X_{1}, P X_{2}, \ldots, P X_{p}=X_{2 p}\right\}$. Then the right hand side of the above equation with the help of (48) can be written as

$$
\begin{aligned}
& g\left(h_{F D_{\theta}}\left(P X_{i}, Z_{r}\right), h_{F D_{\theta}}\left(P X_{i}, Z_{r}\right)\right) \\
&=\csc ^{2} \theta \sum_{r=1}^{q}\left[2 \sum_{i=1}^{2 p}\left(g\left(h\left(P X_{i}, Z_{r}\right), F X_{i}\right)\right)^{2}\right. \\
&\left.\quad+\sum_{i=1}^{2 p} \sum_{s=1, s \neq i}^{2 p}\left(g\left(h\left(P X_{i}, Z_{r}\right), F X_{s}\right)\right)^{2}\right] .
\end{aligned}
$$

From (44), (50), and expression (53), it is clear that equality holds if $h(D, D)=0, h\left(D^{\perp}, D^{\perp}\right)=0, h\left(P X_{i}, Z_{r}\right)$ is normal to $F Z_{t}$ and $h\left(P X_{i}, Z_{r}\right)$ is normal to $F X_{i}$ and $F X_{s}, i, s=$ $1,2, \ldots, 2 p, r, t=1, \ldots, q$, where $i \neq s$ and $r \neq t$.

Remark 10. Since (37) is the key result of the paper which helps to get the inequality in Theorem 9 and moreover (37) is also true for the Kaehler manifolds, hence the results in Theorem 9 are also true for hemi-slant warped product submanifolds of a Kaehler manifold. 
Now we compile some results of [16] and give the following example of a warped product submanifold in $S^{6}$.

Example 11. Let $\left\{e_{0}, e_{i}(1 \leq i \leq 7)\right\}$ be the canonical basis of the Cayley division algebra on $R^{8}$ over $R$ and $R^{7}$ the subspace of $R^{8}$ generated by the purely imaginary Cayley numbers $e_{i}(1 \leq i \leq 7)$. Then

$$
S^{6}=\left\{x_{1} e_{1}+x_{2} e_{2}+\cdots+x_{7} e_{7}: x_{1}^{2}+x_{2}^{2}+\cdots+x_{7}^{2}=1\right\} \subset R^{7}
$$

is a unit sphere admitting a nearly Kaehler structure $(J, g, \bar{\nabla})$ as has been specified earlier. Now suppose that $S^{2}=\{x=$ $\left.\left(x_{2}, x_{4}, x_{6}\right) \in R^{3}: x_{2}^{2}+x_{4}^{2}+x_{6}^{2}=1\right\}$ is a unit 2-sphere. For a real triple $p=\left(p_{1}, p_{2}, p_{3}\right)$ with $p_{1}+p_{2}+p_{3}=0$ and $p_{1} p_{2} p_{3} \neq 0$, define a mapping $\psi_{p}$ of $S^{2} \times R$ to $S^{5} \subset S^{6}$ as follows:

$$
\begin{aligned}
\psi_{p}\left(x_{1}, x_{2}, x_{3}, t\right)= & x_{1}\left(\cos \left(t p_{1}\right) e_{1}+\sin \left(t p_{1}\right) e_{5}\right) \\
& +x-2\left(\cos \left(t p_{2}\right) e_{2}+\sin \left(t p_{2}\right) e_{6}\right) \\
& +x_{3}\left(\cos \left(t p_{3}\right) e_{3}+\sin \left(t p_{3}\right) e_{7}\right)
\end{aligned}
$$

where $x_{1}^{2}+x_{2}^{2}+x_{3}^{2}=1$ and $t \in R$. Then $\psi_{p}$ gives rise to an isometric immersion from warped product Riemannian manifold $S^{2} \times{ }_{f} R$ into $S^{6}$. Moreover, induced warped product metric $\bar{g}$ on $S^{2} \times{ }_{f} R$ is given by

$$
\bar{g}=\pi_{1}^{*} g_{0}+\left(\sum_{i=1}^{3}\left(x_{i} p_{i}\right)^{2}\right) \pi_{2}^{*} d t^{2},
$$

where $\pi_{1}: S^{2} \times{ }_{f} R \rightarrow S^{2}$ and $\pi_{2}: S^{2} \times R \rightarrow R$ are natural projections and $g_{0}$ is the Riemannian metric on $S^{2}$ and from (27) it is evident that warping function $f$ is as follows:

$$
f=\sqrt{\sum_{i=1}^{3}\left(x_{i} p_{i}\right)^{2}} .
$$

\section{Conflict of Interests}

The authors declare that there is no conflict of interests regarding the publication of this paper.

\section{Acknowledgment}

The authors are highly thankful to anonymous referees for their valuable suggestions and comments which have improved the paper.

\section{References}

[1] R. L. Bishop and B. O’Neill, "Manifolds of negative curvature," Transactions of the American Mathematical Society, vol. 145, pp. $1-49,1969$.

[2] S.-T. Hong, "Warped products and black holes," Il Nuovo Cimento della Società Italiana di Fisisca B, vol. 120, no. 10-11, pp. 1227-1234, 2005.
[3] B.-Y. Chen, "Geometry of warped product CR-submanifolds in Kaehler manifolds," Monatshefte für Mathematik, vol. 133, no. 3, pp. 177-195, 2001.

[4] B. Sahin, "Warped product submanifolds of Kaehler manifolds with a slant factor," Annales Polonici Mathematici, vol. 95, no. 3, pp. 207-226, 2009.

[5] S. Uddin and A. Y. M. Chi, "Warped product pseudo-slant submanifolds of nearly Kaehler manifolds," Analele Stiintifice ale Universitatii Ovidius Constanta Seria Matematica, vol. 19, no. 3, pp. 195-204, 2011.

[6] B.-Y. Chen, “CR-submanifolds of a Kaehler manifold. I," Journal of Differential Geometry, vol. 16, no. 2, pp. 305-322, 1981.

[7] B. Sahin, "Non existence of warped product semi-slant submanifolds of Kaehler manifolds," Geometriae Dedicata, vol. 117, pp. 195-202, 2006.

[8] V. A. Khan and K. A. Khan, "Generic warped product submanifolds in nearly Kaehler manifolds," Contributions to Algebra and Geometry, vol. 50, no. 2, pp. 337-352, 2009.

[9] K. Sekigawa, "Some CR-submanifolds in a $S^{6}$-dimensional sphere," Tensor. New Series, vol. 41, no. 1, pp. 13-20, 1984.

[10] T. Fukami and S. Ishihara, "Almost Hermitian structure on $S^{6, "}$ The Tohoku Mathematical Journal, vol. 7, pp. 151-156, 1955.

[11] B.-Y. Chen, "Slant immersions," Bulletin of the Australian Mathematical Society, vol. 41, no. 1, pp. 135-147, 1990.

[12] N. Papaghiuc, "Semi-slant submanifolds of a Kaehlerian manifold," Analele Universitatii "Al. I. Cuza" Lasi, vol. 40, no. 1, pp. 55-61, 1994.

[13] J. L. Cabrerizo, A. Carriazo, L. M. Fernández, and M. Fernández, "Semi-slant submanifolds of a Sasakian manifold," Geometriae Dedicata, vol. 78, no. 2, pp. 183-199, 1999.

[14] A. Carriazo, New Developments in Slant Submanifolds Theory, Narosa Publishing House, New Delhi, India, 2002.

[15] V. A. Khan and M. A. Khan, "Pseudo-slant submanifolds of a Sasakian manifold," Indian Journal of Pure and Applied Mathematics, vol. 38, no. 1, pp. 31-42, 2007.

[16] H. Hashimoto and K. Mashimo, "On some 3-dimensional CR submanifolds in $S^{6}$," Nagoya Mathematical Journal, vol. 156, pp. 171-185, 1999. 


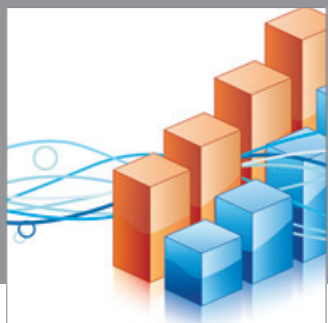

Advances in

Operations Research

mansans

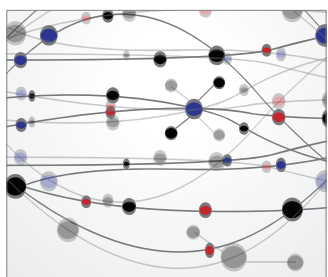

The Scientific World Journal
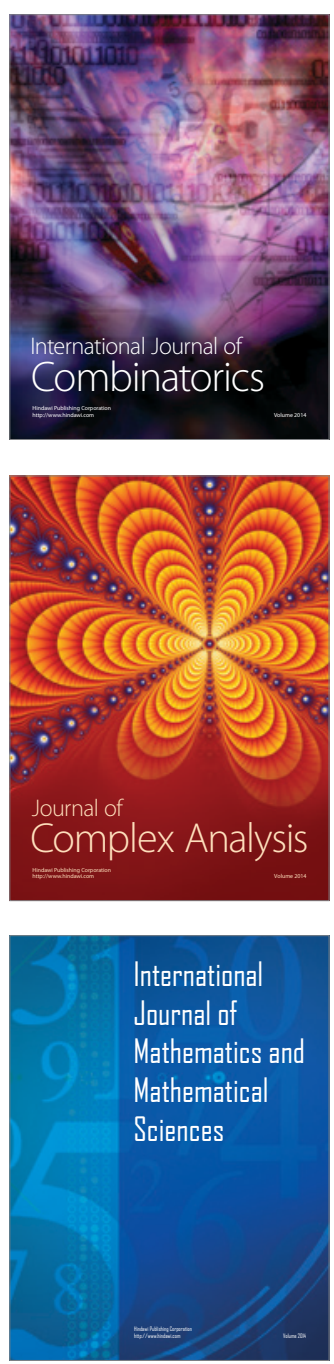
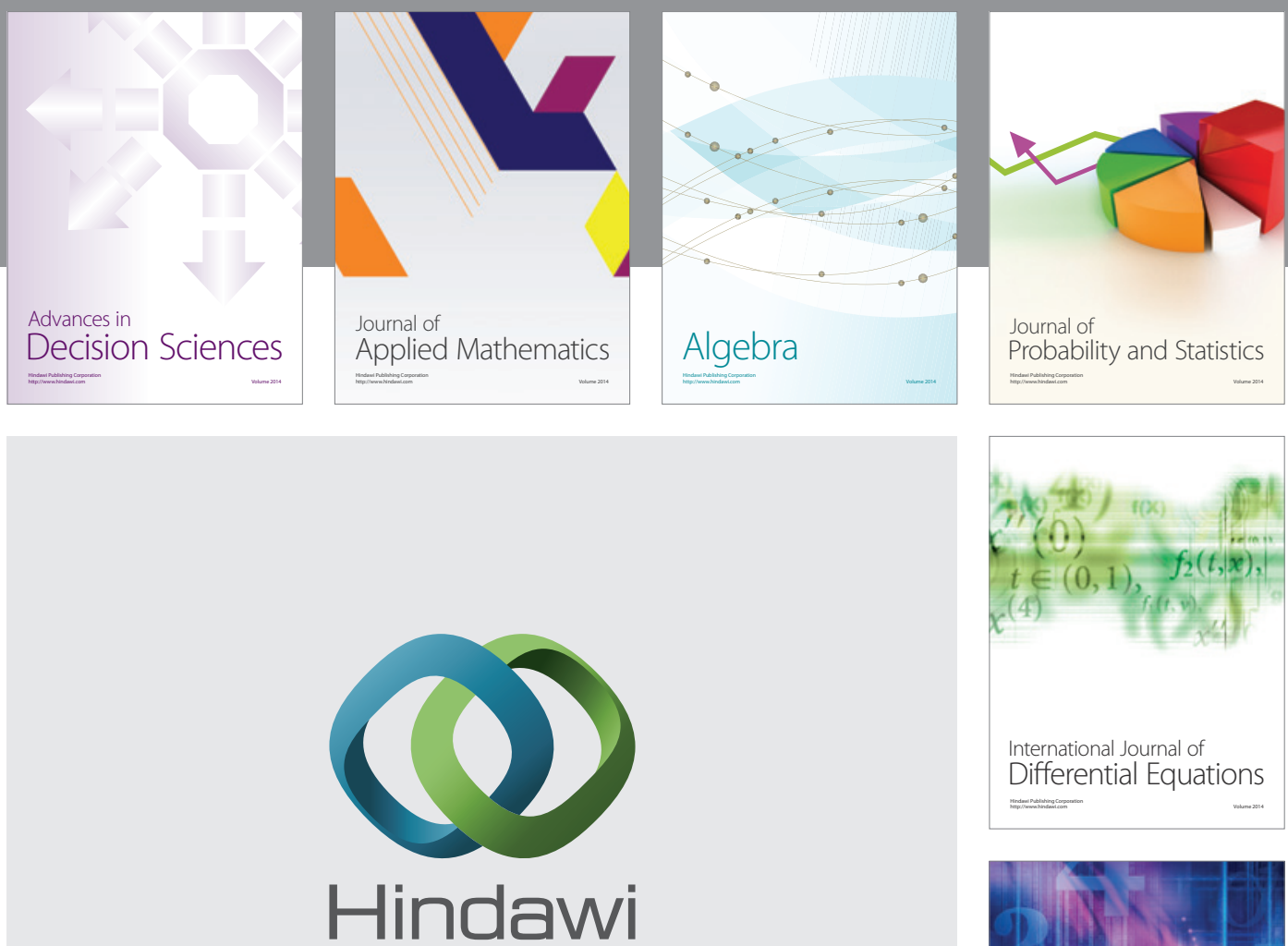

Submit your manuscripts at http://www.hindawi.com
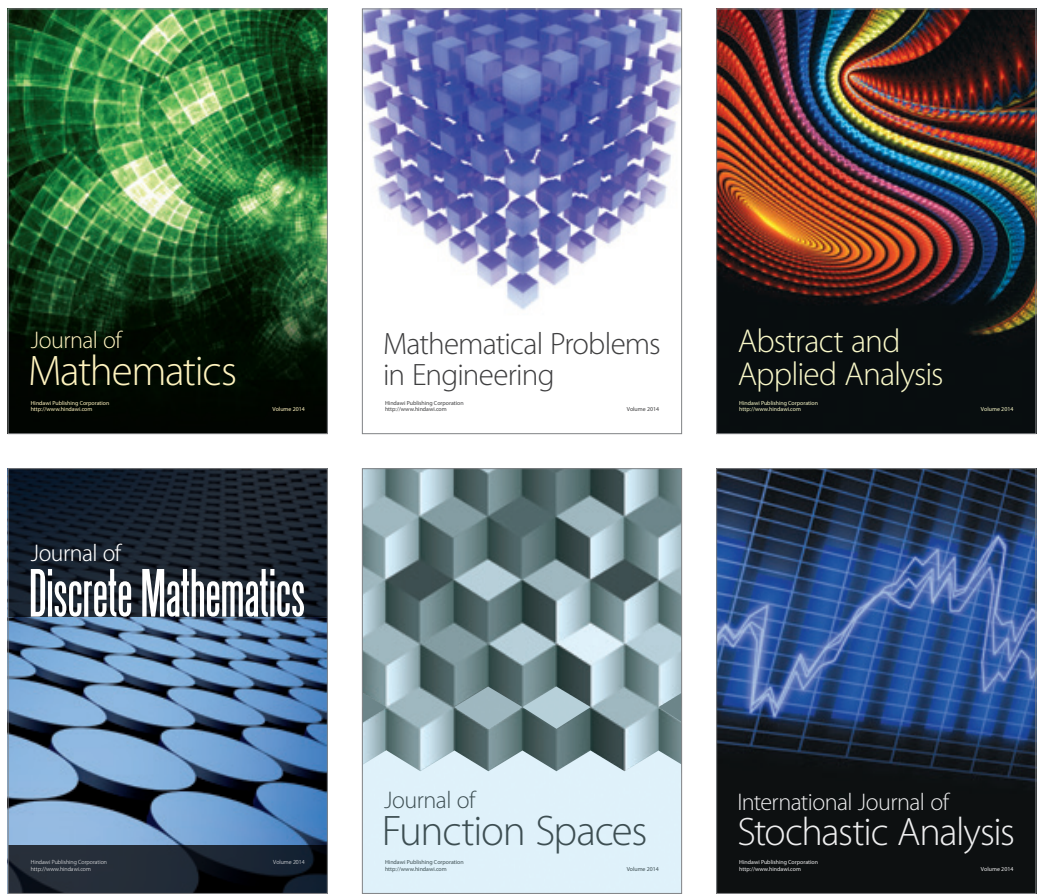

Journal of

Function Spaces

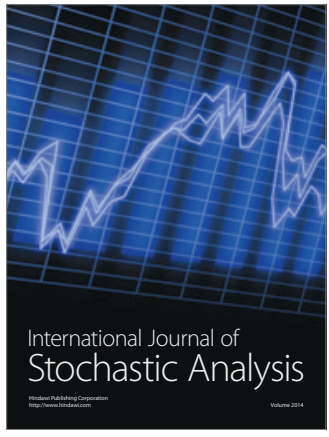

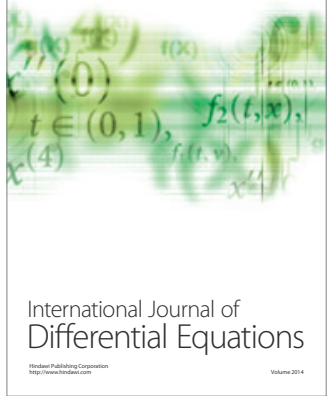
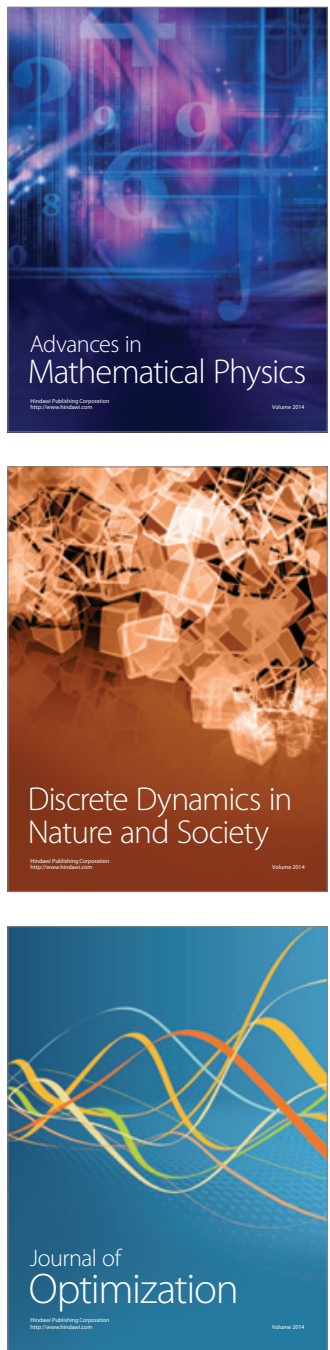